NOTES

\title{
Frustrated Smectic Phase with Unusual Density Modulation along Layer Observed in Main Chain Type of Polymers
}

\author{
Yasukazu NaKata and Junji Watanabe
}

Department of Polymer Chemistry, Tokyo Institute of Technology,

Ookayama, Meguro-ku, Tokyo 152, Japan

(Received September 4, 1996)

\begin{abstract}
KEY WORDS Main Chain Polymer / Alternate Copolymer / Smectic Liquid Crystal / Bilayer / Frustrated Smectic Phase / Ferroelectric Liquid Crystal /
\end{abstract}

During the course of studies on thermotropic mesophases of the main-chain BB- $n$ polyesters, ${ }^{1-16}$

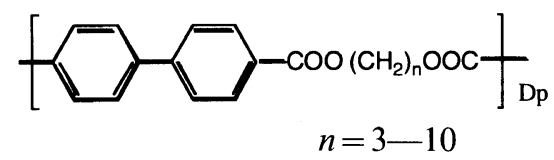

we have found that there are formed two different phases depending on the number of intervening methylene units, $n$. From BB- $n$ with even $n$, a $S_{\mathrm{A}}$ phase is formed in which the axes of both the polymer chain and mesogenic group lie perpendicular to the layers. In contrast, BB- $n$ with odd $n$ formed a $S_{\mathrm{CA}}$ where the polymer chains lie perpendicular to the layer but the mesogenic groups are tilted to the layer normal. The distinct structural feature in the $S_{\mathrm{CA}}$ phase is that the tilt direction of the mesogenic groups is opposite in two neighboring layers. ${ }^{1,2,4}$ Such an odd-even effect on the smectic structures has been explained as resulting from a coupling of the polymeric and mesogenic effects whereby the spatial arrangement of mesogenic groups within a polymer molecule is strongly confined by the conformation of intervening alkylene spacer. ${ }^{16}$ The polymers with odd $n$ tend to assume the conformation with two successive mesogenic groups tilted toward each other, while the mesogenic groups in polymers with even $n$ lie parallel to each other.

In addition to the conformational constraint, another interesting effect can be considered in smectic structure of the main-chain type of polymer with the two kinds of alkylene spacers sequenced in a regularly alternate fashion. ${ }^{9,11}$

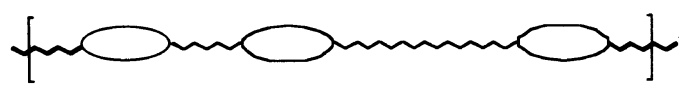

If the two spacers are incompatible sterically, and if there is sufficient lateral attraction between identical spacers of adjacent polymer molecules, segregation into bilayer may occur. Then, four possible types of bilayer structures will arise as a result of a coupling with conformational constraint, as reported previously. ${ }^{9}$ Among these, the bilayer smectic phase of Figure 1a which may be formed from the polymers with two odd numbered spacers is especially interesting since it would be ferroelectric even in achiral system. The space group (Figure 1b) is analogous to a crystallographic $C_{2 \mathrm{v}}$ and spontaneous polarization can be expected to arise along the tilt direction of molecule in a layer. ${ }^{17,18}$

The previous study has been performed to seek this type of ferroelectric smectic liquid crystals in the following main-chain polymers in which two mesogenic biphenyl moieties and two odd-numbered aliphatic chains are sequenced in a regularly alternate fashion. ${ }^{9}$

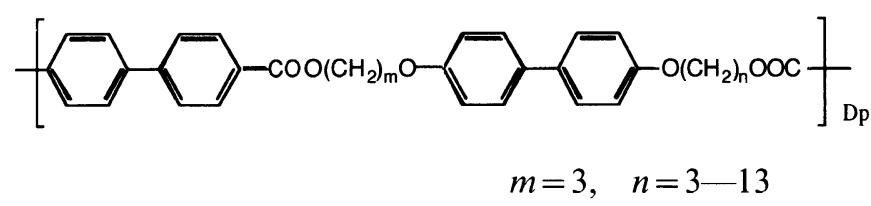

These polymers were designated $\mathrm{BP}-\mathrm{CO}_{3}-\mathrm{O}_{n}$; the aliphatic chain containing three methylene units is connected to the mesogenic cores by two ester linking groups while the other aliphatic chain with a variable odd number of methylene groups $(n)$ is linked to the mesogenic cores by two ether groups. All the $\mathrm{BP}-\mathrm{CO}_{3}-\mathrm{O}_{n}$ polymers with $n=3-13$ have been found to form the smectic phases and two fundamental characteristics of layered structure have been observed as follows. One is that the accommodation of molecules into the layer depends on the difference in lengths of the two aliphatic spacers. If the two spacers have a smaller difference $(\Delta n \leq 6)$ in their lengths, single layer structure is formed as a result of random mixing of the two spacers. In contrast, if there is sufficiently large difference $(\Delta n \geq 8)$, bilayer smectic phase results with a segregation of the two spacers. The second is that the bilayer smectic phase has a frustrated structure with an unusual density modulation in a direction parallel to the layer. The frustrated structure is peculiar and is explained by a two dimensional escape of the spontaneous polarization. ${ }^{9}$ This paper reports some details of structure of the frustrated smectic phase.

\section{Polymers Exhibiting the Frustrated Smectic Phase}

In this study, we have prepared a series of $\mathrm{BP}-\mathrm{CO}_{m}-\mathrm{O}_{n}$ with $m=3-9$ and $n=2-15$; the detailed description of polymer preparation is reported in Ref 11 . By various combinations of $m$ and $n$, we found that the smectic phases with the bilayer character are formed in the polymers with combinations of $m=5, n=2 ; m=7, n=2$; $m=8, n=2 ; m=9, n=2 ; m=3, n=11 ; m=3, n=13$; $m=3, n=15 ; m=5, n=15 .{ }^{19}$ This result shows that a large difference between $m$ and $n$ is necessary for an induction of the bilayer segregation. Further, we found 


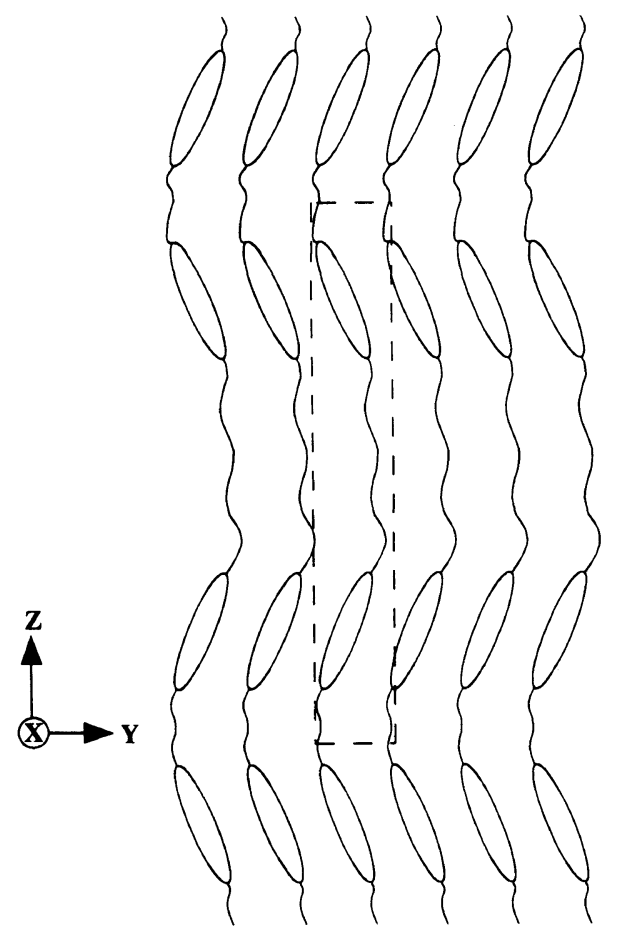

(a)

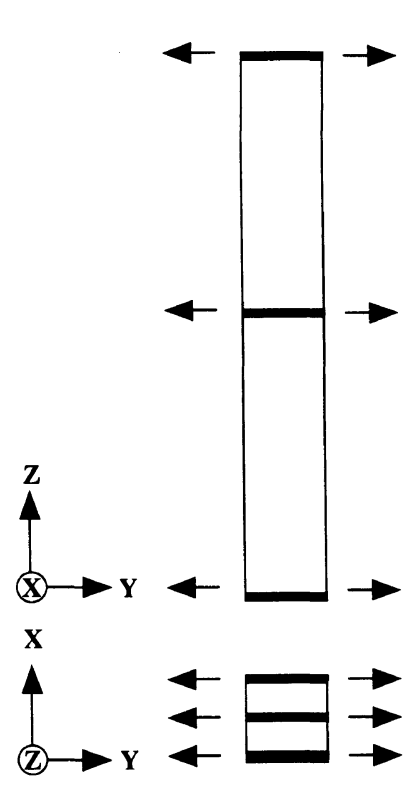

(b)

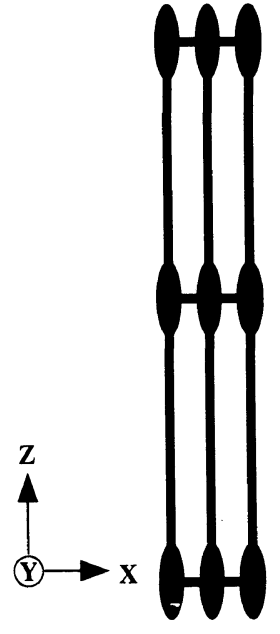

Figure 1. (a) The smectic phase with bilayer modification that may be formed in the main chain polymer with two odd-numbered spacers sequenced in a regularly alternate fashion, and (b) its space-group symmetry.

Table I. Characterization of $\mathrm{BP}-\mathrm{CO}_{m}-\mathrm{O}_{n}$ exhibiting the frustrated smectic phase

\begin{tabular}{|c|c|c|c|c|c|c|}
\hline \multirow{2}{*}{ Polymer } & \multirow{2}{*}{$\eta_{\mathrm{inh}} / \mathrm{dL} \mathrm{g}^{-1 \mathrm{a}}$} & \multicolumn{5}{|c|}{$\begin{array}{l}\text { Transition temperature } /{ }^{\circ} \mathrm{C}^{\mathrm{b}} \\
\text { (Transition enthalpies } / \mathrm{kJ} \mathrm{mol}^{-1} \text { ) }\end{array}$} \\
\hline & & $\mathrm{K}$ & & $\mathrm{Sm}$ & & I \\
\hline $\mathrm{BP}-\mathrm{CO}_{3}-\mathrm{O}_{11}$ & 0.58 & 0 & $145(3.8)$ & 0 & $202(19.2)$ & 0 \\
\hline $\mathrm{BP}-\mathrm{CO}_{3}-\mathrm{O}_{13}$ & 0.37 & 0 & $157(3.8)$ & 0 & $196(23.4)$ & 0 \\
\hline $\mathrm{BP}-\mathrm{CO}_{3}-\mathrm{O}_{15}$ & 0.63 & 0 & $159(4.5)$ & 0 & $183(20.0)$ & $\mathrm{O}$ \\
\hline $\mathrm{BP}-\mathrm{CO}_{5}-\mathrm{O}_{15}$ & 0.28 & 0 & $132(4.9)$ & 0 & $161(16.1)$ & 0 \\
\hline
\end{tabular}

${ }^{\mathrm{a}}$ Inherent viscosities were determined at $25^{\circ} \mathrm{C}$ using $0.5 \mathrm{dL} \mathrm{g}^{-1}$ solutions in a $60 / 40 \mathrm{w} / \mathrm{w}$ mixture of phenol and tetrachloroethane. ${ }^{\mathrm{b}}$ Based on cooling DSC data: $\mathrm{K}=$ crystal; $\mathrm{Sm}=$ smectic; $\mathrm{I}=$ isotropic melt.

that the frustrated smectic phase is formed only from the polymers with odd $m$ and $n$, namely, $\mathrm{BP}-\mathrm{CO}_{3}-\mathrm{O}_{11}, \mathrm{BP}-$ $\mathrm{CO}_{3}-\mathrm{O}_{13}, \mathrm{BP}-\mathrm{CO}_{3}-\mathrm{O}_{15}$ and $\mathrm{BP}-\mathrm{CO}_{5}-\mathrm{O}_{15}$. This means that the odd number combination of $m$ and $n$ is essential for the formation of frustrated smectic phase. Transition temperatures of these four samples are listed in Table I.

\section{$X$-Ray Pattern Characteristic of the Frustrated Smectic Phase}

Figure 2 shows the typical X-ray pattern observed for the frustrated smectic phase of $\mathrm{BP}-\mathrm{CO}_{3}-\mathrm{O}_{15}$ fiber. All the samples exhibit a similar characteristic pattern as can be seen here. One of the characteristics is that the meridional layer reflection is accompanied by four point (off-meridional) reflections. As listed in Table II, the spacing of the first meridional reflection corresponds to half the length of repeat unit of polymer $(L / 2)^{9,11}$ while the off-meridional reflections appear with the height of

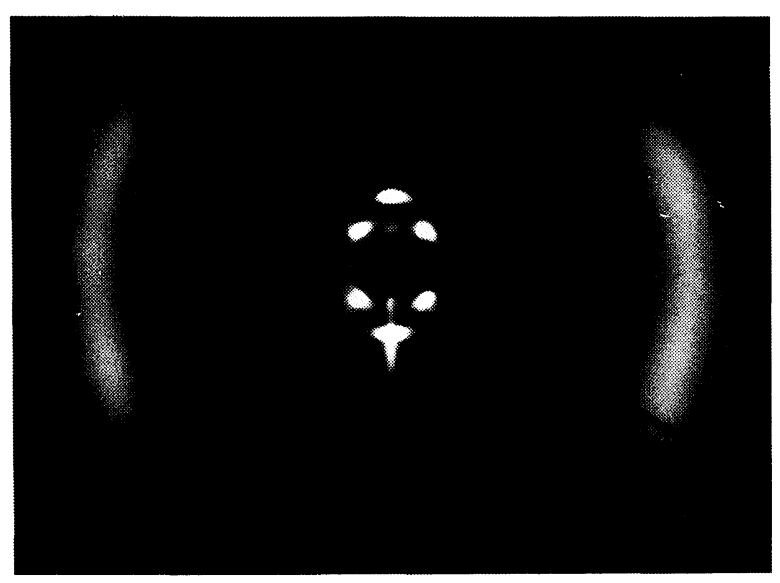

Figure 2. X-Ray diffraction pattern observed for the smectic phase of $\mathrm{BP}-\mathrm{CO}_{3}-\mathrm{O}_{15}$ fiber (at $180^{\circ} \mathrm{C}$ ). The fiber specimen was prepared by pulling up the isotropic melt. The fiber axis is in the vertical direction.

$1 / L$ far from the equator. This dictates that there is a density modulation with a periodicity of $L$ along the layer normal. The X-ray photographs show additionally weak but distinct higher order reflections on meridian and off-meridian, which are also listed in Table II. From the spacing value and diffraction geometry, all of these reflections can be interpreted as $00 l(l=$ even) and $10 l$ $(l=$ odd) reflections based on the two-dimensional rectangular lattice. The lattice parameters are determined as $a=28.5 \AA$ and $c=34.2 \AA$ for $\mathrm{BP}-\mathrm{CO}_{3}-\mathrm{O}_{11}, a=33.8 \AA$ and $c=36.2 \AA$ for $\mathrm{BP}-\mathrm{CO}_{3}-\mathrm{O}_{13}, a=36.9 \AA$ and $c=38.0 \AA$ for $\mathrm{BP}-\mathrm{CO}_{3}-\mathrm{O}_{15}$, and $a=32.5 \AA$ and $c=41.2 \AA$ for $\mathrm{BP}-\mathrm{CO}_{5}-\mathrm{O}_{15}$. Here, the $c$-axis corresponds to the polymer chain axis and its unit length corresponds to the repeat length of polymer. $a$-Axis lies perpendicular to the 
polymer chain. Considering that the averaged diameter of molecule is more or less than $4.5 \AA$, six to eight molecules lie along a-axis in these lattices. The diffuse

Table II. The spacings $(\AA)$ of the inner reflections observed for frustrated smectic phases

\begin{tabular}{cccc}
\hline $\mathrm{BP}-\mathrm{CO}_{3}-\mathrm{O}_{11}$ & $\mathrm{BP}-\mathrm{CO}_{3}-\mathrm{O}_{13}$ & $\mathrm{BP}-\mathrm{CO}_{3}-\mathrm{O}_{15}$ & $\mathrm{BP}-\mathrm{CO}_{5}-\mathrm{O}_{15}$ \\
\hline $21.9(101)$ & $24.7(101)$ & $26.5(101)$ & $25.5(101)$ \\
$17.1(002)$ & $18.0(002)$ & $19.0(002)$ & $20.5(002)$ \\
& & $12.1(103)$ & \\
$8.54(004)$ & $9.04(004)$ & $9.51(004)$ & $10.3(004)$ \\
$6.66(105)$ & & & \\
$5.70(006)$ & $6.02(006)$ & $6.34(006)$ &
\end{tabular}

$($ ), indices of reflections based on two dimensional lattices in the text.

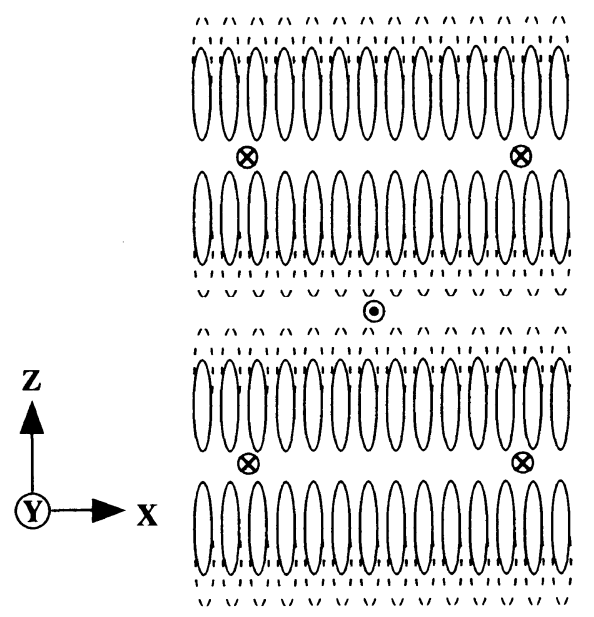

(a) outer reflections are observed above and below the equator. Hence, the mesogenic groups are liquid-like in a layer and their axes are alternately tilted to the layer normal similarly as in the $S_{\mathrm{CA}} \cdot{ }^{4}$ Overall results show that the smectic phase formed here has a bilayer character of Figure 1 although it also has another periodic density modulation in a direction parallel to the layer irrespective of the liquid-like lateral association of mesogenic groups.

\section{Illustration of the Frustrated Smectic Structure}

The characteristic patterns containing the meridional and off-meridional layer reflections are reminiscent for the frustrated smectic phases. ${ }^{20-22}$ These phases were first observed for low molar mass compounds possessing a strong longitudinal dipole such as the derivatives of

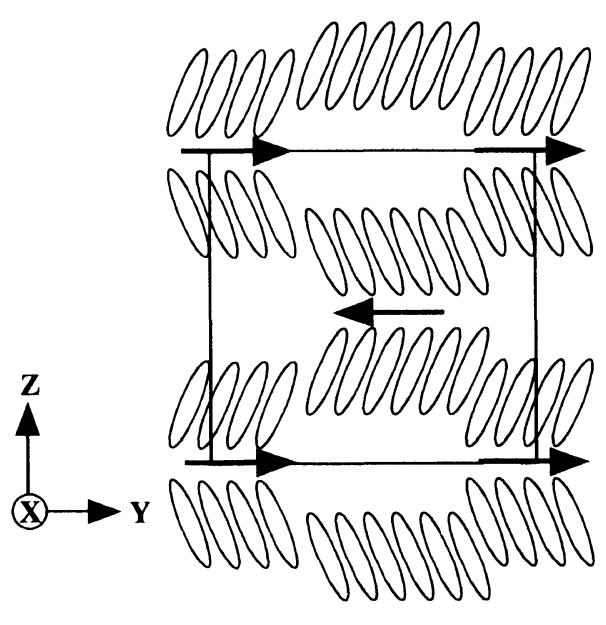

(b)

model A

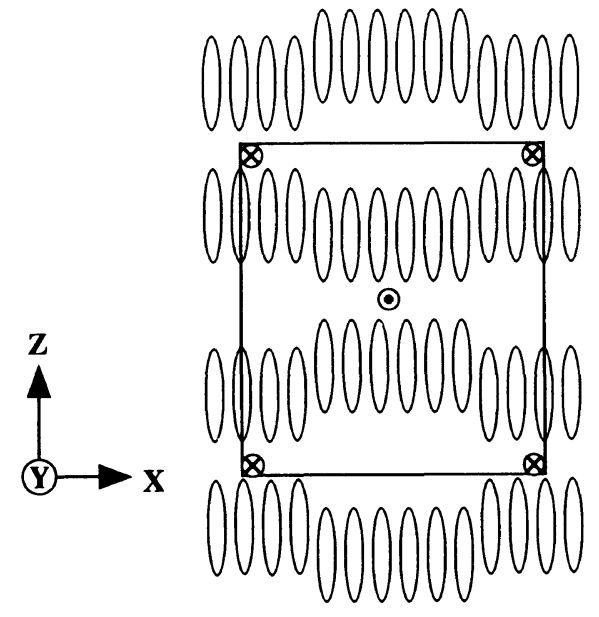

(c)

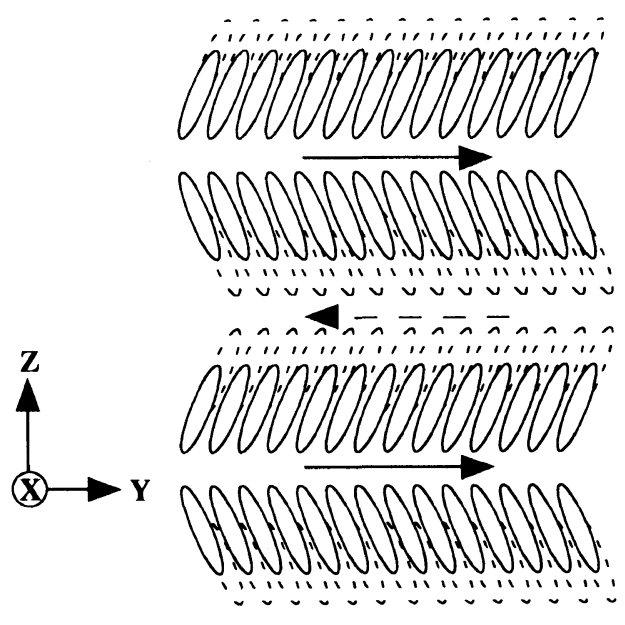

(d)

\section{model B}

Figure 3. Two possible models A and B for the frustrated smectic phase with the unusual density modulation along the layer. In model A, the density modulation arises parallel to the tilt direction of mesogenic groups ( $y$-axis of Figure $1 \mathrm{~b}$ ) while in model B it lies perpendicular to the tilt direction ( $x$-axis). (a) of model A and (c) of model B illustrate the layer structure projected along $x$-axis and (b) of model A and (d) of model B correspond to the layer structure projected along $y$-axis. Solid lines and arrows indicate the two-dimensional rectangular lattice and the spontaneous polarization, respectively (see the text). 
cyanobiphenyl. ${ }^{20}$ The frustrated effect, that is the two dimensional density modulation, arises in such a system because of the incommensurability of two types of characteristic lengths, namely, the molecular length and the pair length, the pair formation being controlled by dipolar forces. ${ }^{21,22}$ Using this concept, we can reach a unique structural model (see Figure 3) in which the bilayer is constructed in a small domain but the polymer chains in adjacent domain slide halfway along the layer normal. In addition to the sliding, the $180^{\circ}$ rotation around their chain axes is required to maintain the tilted orientation of the mesogenic groups, i.e., to satisfy the above X-ray data. In other words, it can be described by a periodic structure of domain walls; in each domain the basic layer structure is the same as the bilayer structure of Figure 1a and its spontaneous polarization is canceled between neighboring domains. This cancela- tion of polarization is considered as a two-dimensional escape of spontaneous polarization and can be attributed to the formation of such a distinct frustrated structure. Observation of a series of reflections with $l=1$ to 5 dictates the highly positional order along the layer normal ( $c$-axis) while the positional order along the layer ( $a$-axis) is low because of the observation of reflections with only $h=1$. This shows that the density modulation along the layer is not necessarily regular. Further, we notice from the lattice parameter ( $a$-axis) that the length of modulation is larger for large differences between $n$ and $m$.

Here, it should be noted that the fundamental bilayer smectic phase of Figure 1a is biaxial. ${ }^{4,9}$ Hence the density modulation along the layer can occur in two possible directions, parallel to the tilt direction and perpendicular to it. Thus, two models of the frustration are proposed

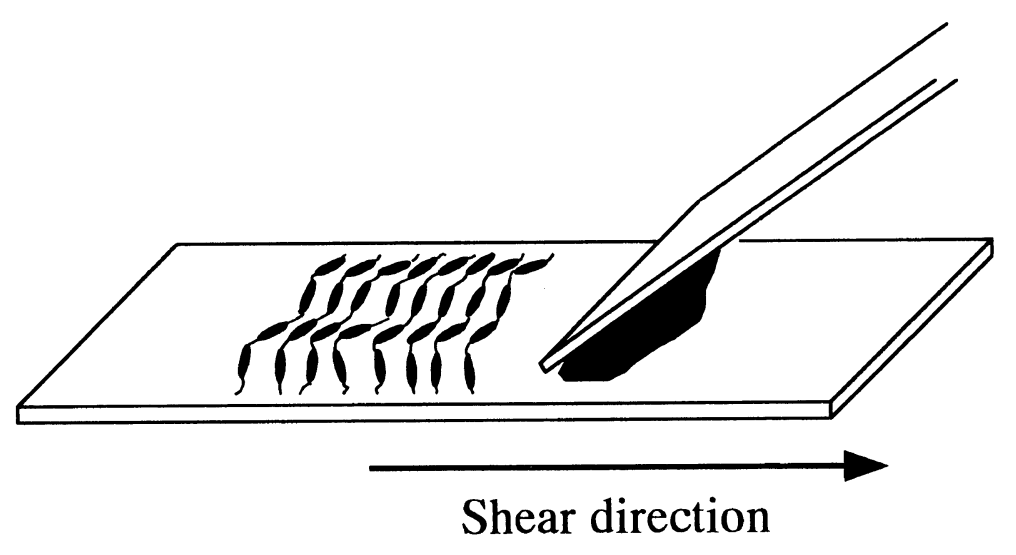

(a)

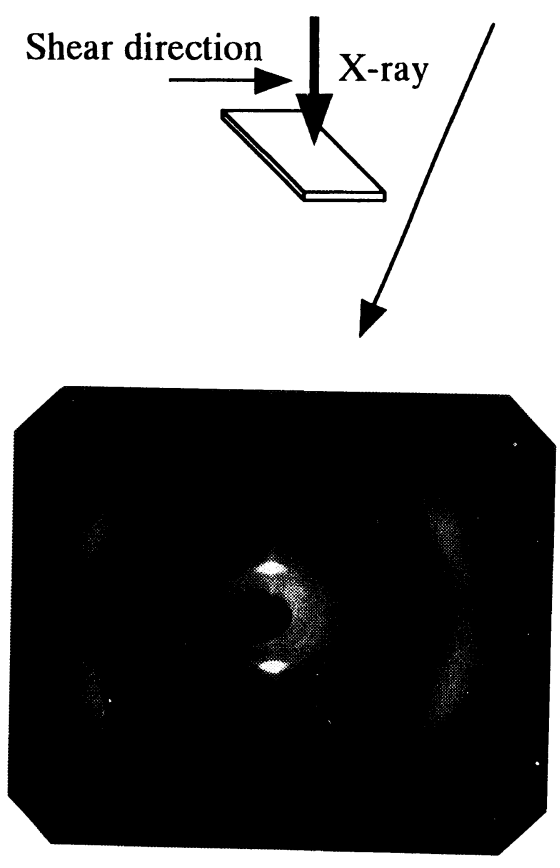

(b)
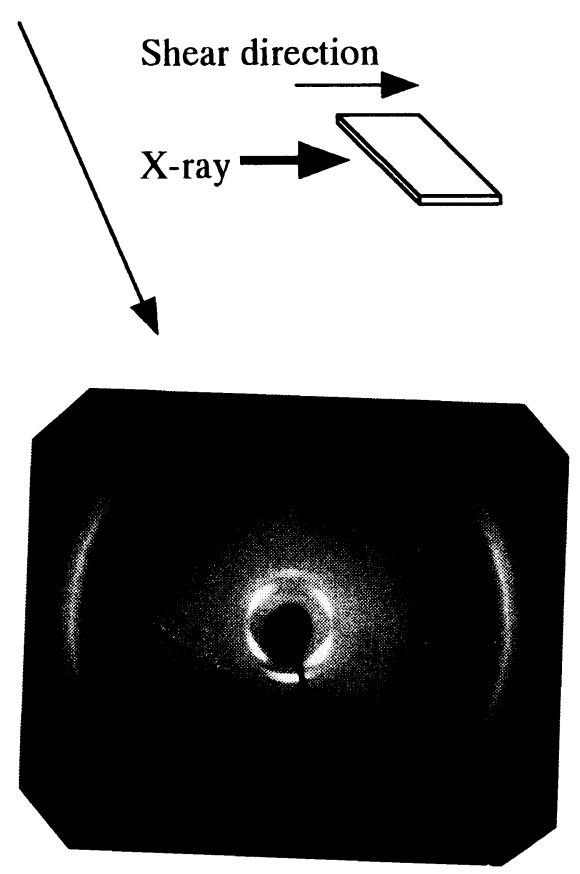

(c)

Figure 4. (a) Illustration of chain orientation in thin film of $\mathrm{BP}-\mathrm{CO}_{3}-\mathrm{O}_{11}$ prepared by shear on a glass plate. Shear results in the chains oriented parallel to the glass (film) surface and perpendicular to the shear direction, and the tilt direction of mesogenic groups lies parallel to the surface. (b) shows the X-ray photograph taken for the smectic film of (a) by the irradiation of X-ray perpendicular to the film surface, while (c) shows the $\mathrm{X}$-ray photograph taken by the irradiation parallel to both the film surface and shear direction. In (c), the film quenched to room temperature was used since an appreciable deformation of films takes place in the smectic temperature region. 
in Figure 3. What is interesting with respect to this point is that shearing the smectic phase produces the preferred uniplanar orientation where the chain orients parallel to the glass surface and perpendicular to the shear direction ${ }^{14}$ and the tilt direction of the mesogenic groups lies parallel to the surface (see Figure 4a). This type of uniplanar orientation is characteristic of a biaxial smectic phase ${ }^{19}$ and the X-ray analysis provides a choice between the two models. Figures $\mathbf{4 b}$ and $\mathbf{4 c}$ show the $\mathrm{X}$ ray photographs taken for such an oriented film; in (b), the X-ray was irradiated perpendicular to the film surface while in (c) it was irradiated parallel to the film surface and perpendicular to the chain axis. A remarkable difference between the two types of irradiation can be seen. The outer broad reflection in (b) is split above and below the equator while that in (c) is placed somewhat around the equator, confirming the preferred orientation mentioned above. Further, the distinct difference can be found with respect to the inner reflections. Photograph (b) shows only the reflections with the indices of $(00 l)$ while photograph (c) includes both the $(00 l)$ and $(10 l)$ reflections. The lack of $(10 l)$ reflections in the former leads to the model B of Figure 3 in which the density modulation along the layer takes place perpendicular to the tilt direction.

In conclusion, bilayer smectic phases were formed from the $\mathrm{BP}-\mathrm{CO}_{m}-\mathrm{O}_{\mathrm{n}}$ polymers with the two flexible spacers of remarkably different lengths. Among these, only the polymers with odd number combinations of $m$ and $n$ formed the frustrated smectic phase in which the unusual density modulation arises along the layer. This frustrated structure can be understood such that the bilayer with a segregation of the two spacers is constructed in a small domain but the polymer chains in adjacent domain slide halfway along the layer normal after a $180^{\circ}$ rotation around their chain axis. The density modulation in this biaxial phase takes place perpendicular to the tilt direction of mesogen groups. Such a frustration is considered as a two-dimensional escape from the strong interaction of the spontaneous polariza- tion in a bilayer structure with $C_{2 \mathrm{v}}$ symmetry. ${ }^{18}$

\section{REFERENCES AND NOTES}

1. J. Watanabe and M. Hayashi, Macromolecules, 21, 278 (1988).

2. J. Watanabe and M. Hayashi, Macromolecules, 22, 4083 (1989).

3. H. Takezoe, A. Fukuda, A. Ikeda, Y. Takanishi, T. Uemoto, H. Iwane, M. Hara, K. Itoh, and J. Watanabe, Ferroelectrics, 122, 167 (1991).

4. J. Watanabe and S. Kinoshita, J. Phys. II (France), 2, 1273 (1992).

5. Y. Takanishi, H. Takezoe, A. Fukuda, J. Watanabe, and H. Komura, J. Mater. Chem., 2, 71 (1992).

6. Y. Takanishi, H. Takezoe, A. Fukuda, and, J. Watanabe, Phys. Rev. B, 45, 7684 (1992).

7. J. Watanabe, H. Komura, and T. Niori, Liquid Crystals, 13, 455 (1993).

8. Y. Nakata and J. Watanabe, J. Mater. Chem., 4, 578 (1994).

9. Y. Nakata, K. Shimizu, and J. Watanabe, J. Phys. II (France), 4, 581(1994).

10. J. Watanabe, M. Hayashi, A. Morita, and T. Niori, Mol. Cryst. Liq. Cryst., 254, 221 (1994).

11. Y. Nakata and J. Watanabe, High Perform. Polym., 7, 377 (1995).

12. J. Watanabe, M. Hayashi, A. Atsushi, and M. Tokita, Macromolecules, 28, 8073 (1995).

13. J. Watanabe, T. Niori, and S. Adachi, Liquid Crystals, 19, 139 (1995).

14. M. Tokita, T. Takahashi, M. Hayashi, and J. Watanabe, Macromolecules, 29, 1345 (1996).

15. J. Watanabe, M. Hayashi, and M. Tokita, Reactive \& Functional Polymers, 30, 191 (1996).

16. J. Watanabe, M. Hayashi, Y. Nakata, T. Niori, and M. Tokita, Prog. Polym. Sci., in press.

17. International Table for X-Ray Crystallography," Vol. I, Kynoch Press, Birmingham, 1959, p. 208.

18. In practice, we have observed the spontaneous polarizability in the achiral smectic phase with $C_{2 v}$ symmetry which is formed from the banana-shaped molecules; T. Niori, T. Sekine, J. Watanabe, T. Furukawa, and H. Takezoe, J. Mater. Chem., 6, 1231 (1996)

19. Y. Nakata and J. Watanabe, to be published.

20. G. Sigaud, F. Hardouin, M. F. Achard, and A. M. Levelut, J. Phys. (France), 42, 107 (1981).

21. J. Prost and P. Barois, J. Chim. Phys., 80, 65 (1983).

22. J. Prost, Adv. Phys., 33, 1 (1984). 